\title{
A Human-in-the-Loop Investigation of Multi-Sector Planning Operations for the NextGen Mid-Term
}

\author{
Nancy Smith ${ }^{\mathrm{a}}$ \\ NASA Ames Research Center, Moffett Field, CA, 94035 \\ Paul U. Lee ${ }^{\mathrm{b}}$, Thomas Prevot ${ }^{\mathrm{c}}$, Connie Brasil ${ }^{\mathrm{d}}$, Jeff Homola $^{\mathrm{d}}{ }^{\mathrm{d}}$ Angela Kessell $^{\mathrm{b}}$, Hwasoo Lee $^{\mathrm{d}}$,
Matt Mainini $^{\mathrm{d}}{ }^{\text {, Joey Mercer }}{ }^{\mathrm{d}}$
SJSU / NASA Ames Research Center, Moffett Field, CA, 94035
}

\begin{abstract}
A human-in-the-loop simulation was conducted to evaluate a concept for introducing multi-sector trajectory planning operations into en route air traffic facilities in the NextGen Mid-Term timeframe. Multi-sector planning tools and procedures for local area traffic flow management were developed, and then tested using two different service provider team configurations. In one condition, local area flow planning was performed by the traffic management coordinator and area supervisor. A second condition added a new, dedicated multi-sector planner position to the planning team. A set of eight convective weather and traffic load scenarios was used to evaluate the operational feasibility, potential benefits, and tool performance requirements of each condition. Significant improvements in weather avoidance and controller workload were observed in the multi-sector planner condition but no significant improvement was observed in user efficiency. Results indicate that multisector planning operations are effective and feasible in either team configuration.
\end{abstract}

\section{Introduction}

Air traffic in the United States National Airspace System (NAS) is expected to increase significantly in the coming decades. In today's system, delays are caused when demand exceeds capacity for a given resource, such as an airport or region of airspace, or due to weather that forces the closure of airspace or routes. Despite the recent economic downturn and the associated curtailment in traffic growth, system delays continue, and air traffic is still predicted to grow and put increasing strains on the system as the economy recovers. High-end estimates indicate that by the year 2025 total passenger enplanements may more than double and total aircraft operations may triple in comparison to current traffic levels ${ }^{1}$.

Several developments in the technology supporting air traffic management in the NAS - including digital data communication among controllers and between controllers and aircraft, improved positioning accuracy for flight operations using satellite navigation, improved automation for conflict prediction and resolution, and sector complexity assessment - enable increasing flexibility in the management of aircraft trajectories and reduce the dependency on fixed route structures. These improvements will become more widely available within the Next Generation Air Transportation System (NextGen) Mid-Term timeframe, providing an opportunity to develop new functional and organizational operations that could improve routing options within and around congested airspace.

Timelier access to better information about NAS operations will also provide earlier prediction of resource constraints or disruptive events, enabling a shift towards more strategic and less tactical traffic management for earlier, more efficient solutions when aircraft re-routing is required.

The MSP (Multi-Sector Planner) concept has been explored in both Europe and the United States since the mid1990s, although proposed roles and responsibilities for the new position have varied depending on the operational context, available technology, and perceived need ${ }^{2-6}$. In an earlier FAA-sponsored research effort, two alternative

\footnotetext{
${ }^{a}$ Research Psychologist, Human Systems Integration, MS 262-4.

${ }^{\mathrm{b}}$ Senior Research Associate, Human Systems Integration, MS 262-4.

${ }^{c}$ Senior Research Engineer, Human Systems Integration, MS 262-4.

${ }^{\mathrm{d}}$ Research Associate, Human Systems Integration, MS 262-4.
} 
MSP concepts were developed and compared for feasibility and effectiveness. In one proposed concept, the MSP worked from a remote location, acting as a radar associate controller supporting several sectors' operations. An alternative concept defined the MSP as a local area flow planner, responsible for managing sector load and complexity by selectively modifying the trajectories of individual aircraft. A human-in-the-loop (HITL) simulation was conducted in 2006 to compare each of these MSP concepts to a 'No-MSP' baseline condition. Results indicated that the area flow planner concept was preferred ${ }^{7-8}$. The 2006 study focused on concept feasibility and benefits at the sector level, exploring the interactions between MSP and radar controllers, and evaluating MSP effectiveness in supporting sector operations. Follow-on research was recommended to further define the position's tool requirements and its relationship to other positions within the en route facility.

This recommended research began in 2008, expanding the area flow planner MSP concept in the following three areas:

1. defining MSP roles and responsibilities within the air navigation service provider (ANSP) team, along with any corresponding changes to other team positions (traffic manager, area supervisor and radar controller);

2. developing communication and coordination procedures, and communication infrastructure requirements for MSP operations;

3. developing an integrated set of MSP decision support tools for situation assessment, trajectory/flow manipulation, and plan coordination.

The 2009 HITL simulation described in this paper tested this concept, evaluating the performance of multisector planning operations with and without the introduction of a new MSP position.

\section{Experiment}

\section{A. Concept and Objectives}

\section{The Multi-Sector Planner concept}

The concept for multi-sector planning investigated in this simulation is a process for solving local area flowrelated problems by selectively modifying trajectories of one or more aircraft. Key objectives of multi-sector planning operations are to increase efficiency and reduce delays, and to ensure that sectors do not exceed complexity thresholds. The MSP can help implement traffic management initiatives and redistribute traffic to manage complexity and demand within the sectors that he/she oversees. The MSP operates in the 30-60 minute event horizon, bridging the gap between the close tactical focus of the en route sector controller and the more strategic focus of traffic management. To perform these operations the MSPs need automation tools that allow them to evaluate the local traffic situation and sector load, and to develop and coordinate trajectory change proposals with front line managers, sector team members, local traffic managers, and adjacent MSPs.

\section{Simulation objectives}

The MSP concept initially assumed that a dedicated position would be responsible for managing this activity within a particular multi-sector region of airspace. An alternative approach that would integrate these functions into the existing ANSP team framework offered a potentially simpler and more flexible means for introducing MSP operations. The objectives for this simulation were to evaluate the feasibility and potential benefits of the MSP concept with and without introducing a new position. Tool performance requirements to enable situation assessment, flow and trajectory trial plan development, as well as communication and coordination for multi-sector planning operations were also a key objective of the simulation, and a companion paper addresses findings in this area ${ }^{9}$.

\section{B. Design}

\section{Participants and Airspace}

The experiment was conducted in two separate two-week sessions. Each session included ten participants from FAA en route air traffic facilities: four certified air traffic controllers, one traffic management coordinator (TMC), one front line manager, and four front line managers or traffic management supervisors who had experience in both positions. Twelve recently retired controllers, acting as confederates, staffed the remaining positions. Figure 1 shows the test airspace with participant and confederate staffed positions. 


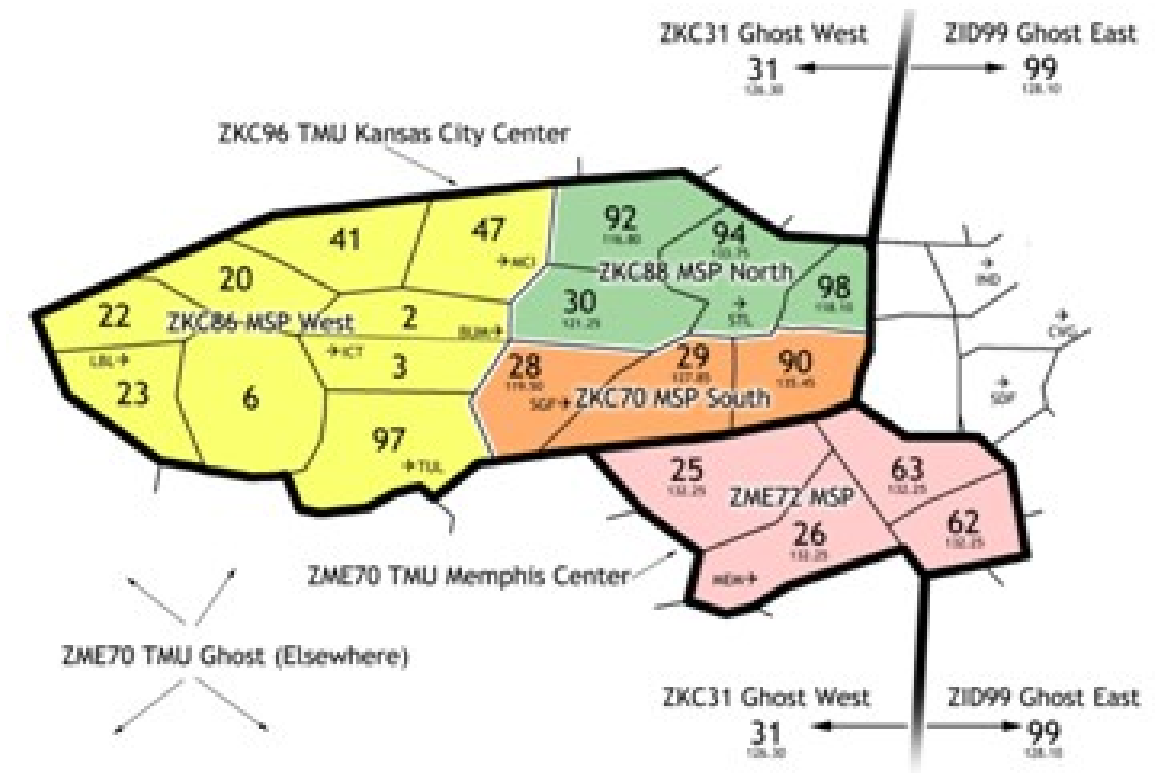

7 ZKC East Test Sectors $(28,29,30,90,92,94,98)$

- Participants: ZKC TMC; ZKC East area supervisor; 4 test sector controllers; MSPs North and MSP South

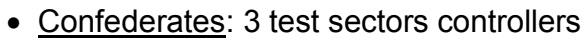

Simulation Positions for Surrounding Airspace

- Participants: MSP West (ZKC) and MSP ZME

- Confederates: ZME / Ghost TMC; Supervisor (ZME, ZKC West and Ghost airspace); 3 multi-sector controllers (ZKC West, ZME and Ghost airspace); 10 pseudo-pilots

Figure 1: Test airspace (top) and list of staffed positions (bottom)

\section{Test conditions}

The two test conditions represented two different air navigation service provider (ANSP) team configurations. Participant teams in the "No-MSP" condition included a traffic management coordinator for Kansas City Center (ZKC), a front-line supervisor responsible for the seven high altitude test sectors on the eastern side of ZKC, and seven test sector controllers (four current and three retired en route controllers). The "MSP" condition added four MSPs to the team. MSP areas of responsibility are color coded in Figure 1.

\section{Scenarios}

There were eight different traffic scenarios. Each scenario was run once in each condition, for a total of sixteen test runs. Four of the scenarios presented a traffic load problem with sector load imbalances within the test airspace at 1.5 times current day traffic levels. Four weather scenarios subjected aircraft and controllers to dynamically changing convective weather situations at current day traffic levels. Each scenario was intended to be unworkable by the sector controllers without some form of upstream intervention or local area flow management.

\section{Simulation environment}

Each run was 75 minutes long with over 1000 aircraft "flown" by automated agents and ten general aviation and corporate pilots. Since a primary focus of this study was on coordination and cooperation within the ANSP team using automation and voice, all trajectory changes were coordinated via land-lines and/or ground-ground digital data communications. Advanced tools were provided for tactical controllers, integrating air-ground data communications for uplinking clearances, weather-penetration and conflict detection algorithms, and trial planning automation that provided weather and conflict resolution advisories.

New tools were also provided for the MSP, TMC and supervisor stations to support flow-based situation assessment, and manipulation and coordination of trajectories for one or several aircraft. Notable among these were 
real-time traffic load and complexity predictions that were integrated with a multi-aircraft trial planning function so that "what if" feedback could be presented in complexity tables and graphs. Figure 2 shows an MSP workstation with these integrated tools, and a close-up view of the Sector Load Table and Load Graphs.
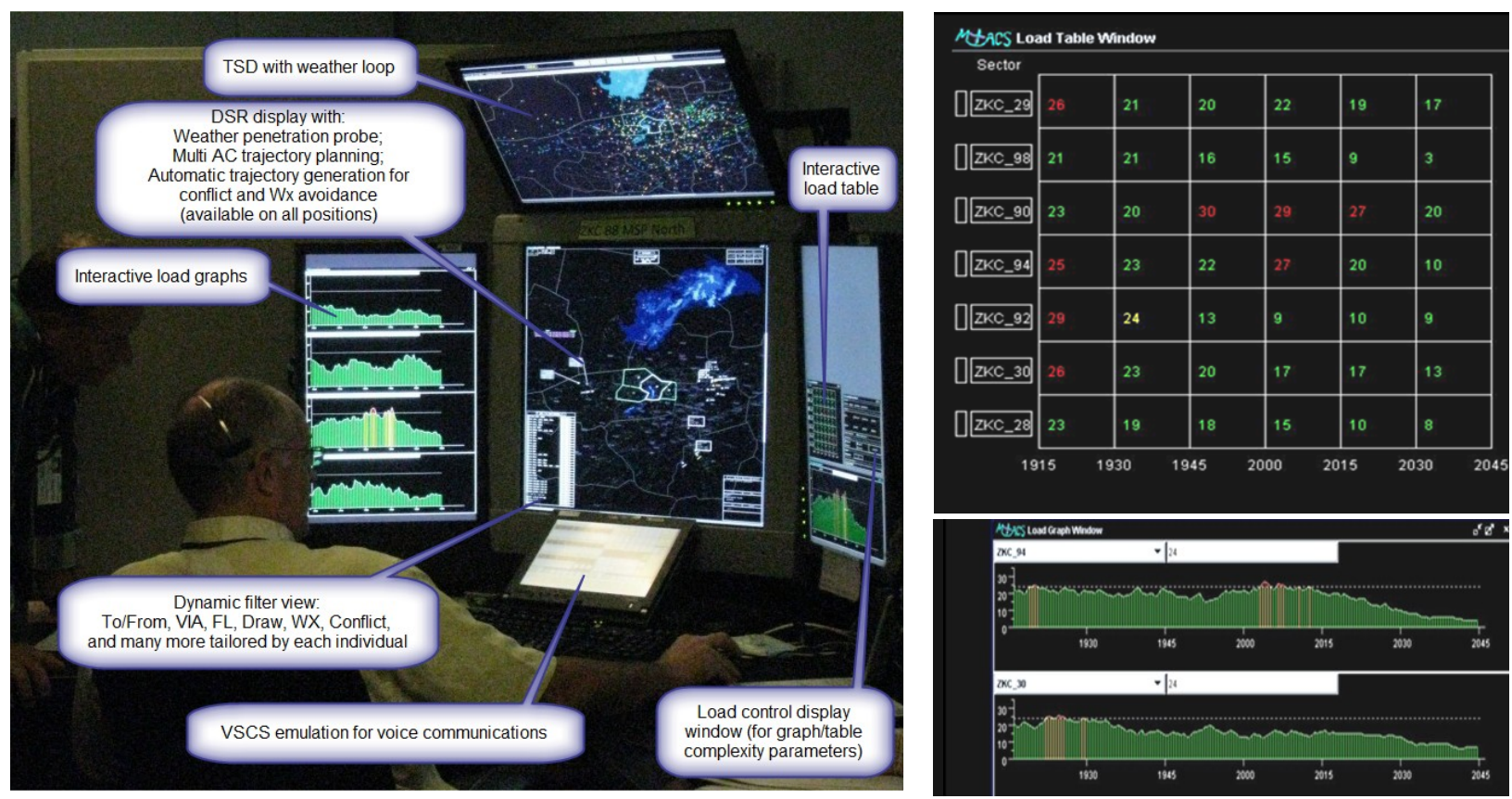

Figure 2: MSP workstation, with situation assessment and flow management tools (left); Load Table (upper right) that shows predicted complexity, aircraft count and/or other measures in 15 minute increments for sectors of interest; Load Graphs (lower right) that show predicted sector loads in 1-minute increments

\section{Roles and responsibilities}

The planning teams were responsible for evaluating the local traffic situation, then developing and coordinating trajectory-based solutions as necessary to maintain efficient local operations and keep controller task load at an acceptable level. In the MSP condition, the planning team for the seven test sectors consisted of the one TMC, one supervisor, and the two MSPs. The TMC, supervisor and MSPs used the situation assessment tools to monitor their area of responsibility. Each worked within a different look-ahead horizon. The supervisor focused on the immediate situation (from "now" through 30 minutes into the future). MSPs were responsible for working withinthe 30-60 minute timeframe but with a focus on the 30-45 minutes time horizon. The TMC addressed the worked beyond 45 minutes. In the No-MSP condition, the TMC assumed responsibility for the 30 minutes time horizon and beyond. When a problem was identified, the TMC and/or MSPs could use interactive features of the Load Table, Load Graphs and traffic display filters to identify aircraft that contributed to the problem, and trial planning tools to selectively plan reroutes for one or several of those aircraft to solve it. Trial plan solutions were coordinated among the planning team using land-lines and ground-ground data communication. The TMC was also responsible for high-level plan coordination and initiating coordination with other facilities as needed. Approved plans were sent as coordinated clearance messages to the controller, who would review each message, then send it as an 'uplink' clearance (UC) to the aircraft via data communications if it was acceptable. If a proposed trajectory change occurred more than 30 minutes in the future, the TMC and MSPs had the option of bypassing the controller and sending the UC message directly to the aircraft.

\section{Metrics}

Experiment metrics included:

- Workload and task load: sector count and complexity; self-reported workload ratings, recorded at 3 minute intervals throughout the run; post-run questionnaire feedback;

- Safety: number of conflicts, time remaining until separation loss at point of resolution; separation violations; number of aircraft that penetrated weather (with duration and severity level);

- System and flight efficiency: flight delay and flight path length compared to original flight trajectory; 
- Communications: voice communication metrics (count, duration, participants and content for simulated radio and land-line communications); digital communication metrics (count, sender, recipient, content and outcome of plan coordination, clearance coordination, and uplink clearance data communications messages);

- Acceptability of operational concept: Post-run and post-simulation questionnaires; debrief discussions;

- Tool performance: Usage and user ratings of automation tools.

"Open Loop" results were obtained for all eight traffic scenarios by running each scenario with no controller input (i.e., "open loop"). Each aircraft entered the Open Loop run at its appropriate time and flew its default flight trajectory without interruption. Open Loop logs of sector count, traffic complexity, weather penetration events and conflicts were then used to represent the problems that the ANSP team were tasked to address, providing a baseline for comparison to support analysis of system effectiveness in each test condition.

\section{Results}

Key results presented in this section are organized into three broad categories. Operational outcome metrics measure how well the ANSP system performed in each condition. Process metrics indicate differences in team performance between conditions, and how those differences may have contributed to different observed outcomes. Participant feedback provides insights into operational feasibility, scope and possible location of multi-sector planning operations. Results related to tool performance are presented in two companion papers. ${ }^{9-10}$

Although the experiment consisted of two sessions, each with a different set of participants, observations made during Session 1 suggested changes in training and procedures could improve the quality of data obtained in Session 2. For simplicity, only Session 2 data is presented in this paper.

\section{A. Outcome Metrics}

Outcome metrics include controller workload, sector traffic load, conflicts, weather penetration events, and flight efficiency (path length changes). Note that these measures reflect the combined performance of both planning and air traffic control operations to varying degrees. Broadly speaking, one of the key outcomes of successful planner complexity and load management is the reduction of the controllers' task load, enabling them to more safely and efficiently control aircraft within their sectors.

\section{Controller workload}

During each simulation run, sector controllers were prompted every three minutes to report their current workload while controlling traffic. They rated their workload on a 1 to 6 scale where ratings of 1 and 2 were considered to be low workload, ratings of 3 and 4 were considered to be medium workload, and ratings of 5 and 6 were considered to be high workload. The resulting in-trial workload ratings were averaged across the sectors and compared between the MSP and No-MSP conditions.

A repeated measures ANOVA was performed on the mean in-trial workload ratings given from 30 minutes into the run until the end of the run (75 minutes into the run). Results showed a small but significant overall reduction in workload in the MSP condition compared to the No-MSP condition $(\mathrm{F}(1,6)=6.01 ; p<0.05)$ but the workload reduction was modest $\left(\mathrm{M}_{\mathrm{No}-\mathrm{MSP}}=3.09\right.$ vs. $\left.\mathrm{M}_{\mathrm{MSP}}=2.82\right)$ and the mean workload was well within a moderate range for both conditions (Figure 3). There was no significant workload difference between the scenario types, Weather or Traffic Load.

Controller participants were asked how acceptable the workload was in their respective sector after each run. Controller workload was rated as more acceptable in the MSP condition than in the No-MSP condition $(F(1,6)=$ $13.5 ; p<0.01)$. On average, the acceptability of the workload in the MSP condition was higher $\left(\mathrm{M}_{\mathrm{MSP}}=4.87\right)$ than in the No-MSP $\left(\mathrm{M}_{\mathrm{No}-\mathrm{MSP}}=4.41\right)$ condition, but in both conditions workload was rated in the moderate to high acceptability range (Figure 4). There was no significant difference across the sectors or scenario types.

The workload acceptability results show that not only was workload reduced during the MSP condition, the reduced workload was more acceptable to the controllers, suggesting that controller workload was managed better in the MSP condition than in the No-MSP condition. 


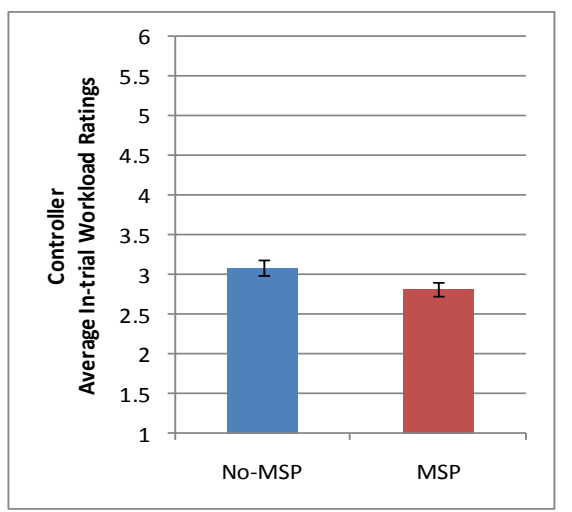

Figure 3: Controllers' mean workload ratings

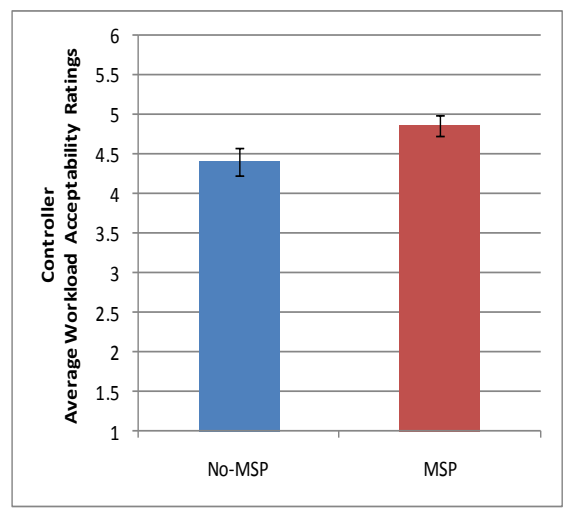

Figure 4: Controllers' mean workload acceptability ratings

\section{Aircraft count}

The workload results showed that controller workload was reduced during the MSP condition for both Traffic and Weather scenarios and the resulting workload was more acceptable to the controllers (see Figures 3 and 4). However, one of the potential reasons for the workload reduction may be due to a reduction in aircraft count. In order to examine the impact of the sector aircraft count on the workload, the aircraft count data was analyzed sideby-side with the workload data. The aircraft count was examined for every minute of simulation run time and the count in MSP and No-MSP conditions were compared to each other as well as the "Open Loop" aircraft counts.

For the weather scenarios, there was little or no reduction of traffic demand for MSP and No-MSP conditions compared to the Open Loop data, suggesting that the aircraft did not need to be rerouted out of the test sectors to manage the traffic complexity in the Weather scenarios. There was also little difference in the aircraft count between MSP and No-MSP conditions, especially in the weather-impacted sectors, suggesting that the workload difference can be attributed to factors other than lower aircraft count.

In the traffic load scenarios, there was a reduction in aircraft count for MSP and No-MSP conditions compared to the Open Loop data, suggesting that the aircraft were rerouted out of the test sectors to manage the traffic load/complexity (see Figure 5). In addition, there was also a small reduction in aircraft count in the MSP condition compared to No-MSP condition, suggesting that the reduced controller workload may have been due to the aircraft being rerouted out of the test sectors (see Figure 6).

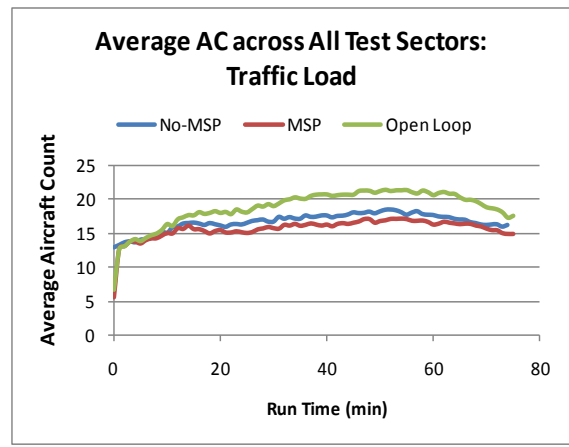

Figure 5: Average aircraft count over time, across all test sectors, Traffic Load runs

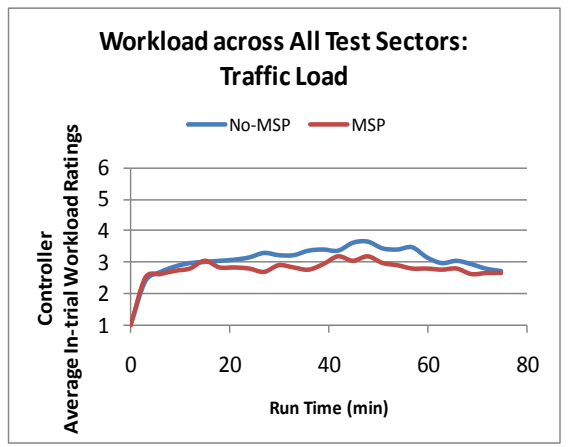

Figure 6: Average controller workload over time, averaged across all test sectors, Traffic Load runs

However, the data from individual sectors suggest that workload reduction could not have been solely due to the reduced aircraft count. For example, Figure 7 shows the average aircraft count for MSP, No-MSP, and Open Loop runs for sector ZKC90. In this figure, the aircraft count showed little difference between the MSP and No-MSP conditions (red vs. blue lines). However, Figure 8, which plots the average workload for the same sector ZKC90, shows a large reduction in workload for the MSP condition compared to the No-MSP condition, suggesting that the workload reduction was due to factors other than aircraft count. 


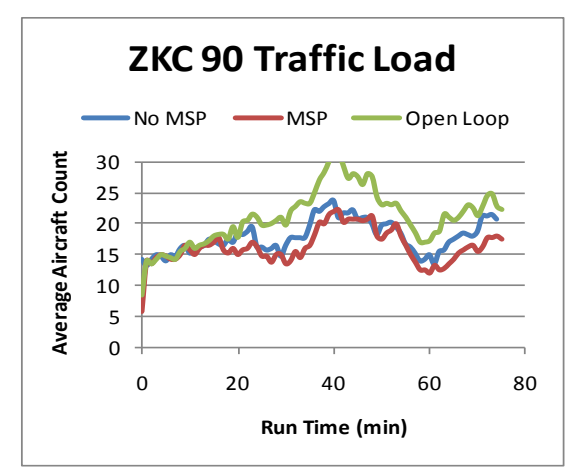

Figure 7: Average aircraft count over time in sector ZKC90, Traffic Load runs

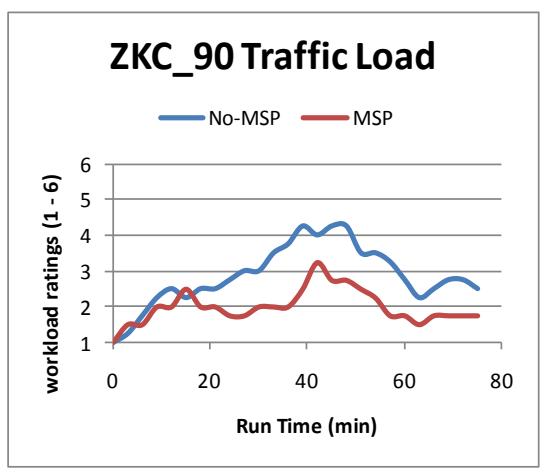

Figure 8: Average controller workload over time in sector ZKC90, Traffic Load runs

\section{Test-sector conflicts}

A unique conflict was defined as a new aircraft pair that was predicted to be in conflict, typically within twelve minutes, by a conflict probe tool. The number of unique conflicts in both test conditions was reduced compared to the Open Loop results, suggesting that the TMCs and/or MSPs efforts to modify aircraft trajectories to resolve traffic overload or weather situations also reduced the number of conflicts in the process (Figure 9). When the MSP and the No-MSP conditions were compared to each other, the MSP condition had consistently fewer conflicts per run than the No-MSP condition in both the weather and traffic load scenarios, although paired t-tests comparing the two conditions were only marginally significant for the traffic load scenarios $\left(\mathrm{M}_{\mathrm{MSP}}=103.00, \mathrm{M}_{\mathrm{No}} \mathrm{MSP}=118.75\right.$; $\mathrm{t}(3)=2.78, \mathrm{p}<0.07)$ and the weather scenarios $\left(\mathrm{M}_{\mathrm{MSP})}=56.50, \mathrm{M}_{\mathrm{No} \text { MSP }}=67.75 ; \mathrm{t}(3)=2.42, \mathrm{p}<0.10\right)$. The marginal significance is likely due to low sample size $(\mathrm{N}=4)$ and a larger sample size would likely have shown a significant reduction in the number of conflicts in the MSP condition.. An additional analysis compared the average minimum times to loss of separation for each conflict pair and found the same trend: conflicts were resolved slightly earlier in the MSP condition, though differences were not significant (see Figure 10).

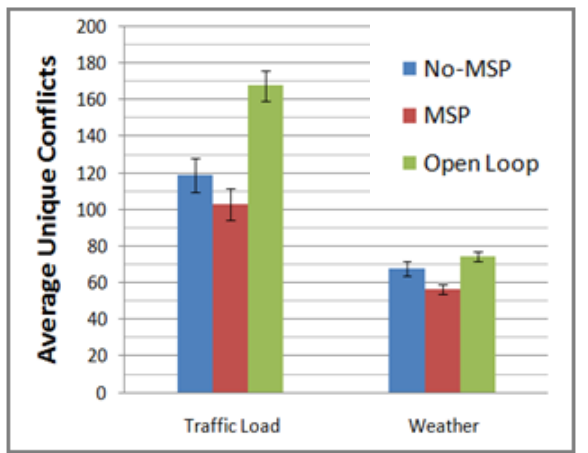

Figure 9: Test sector conflict count, sorted by condition and scenario type

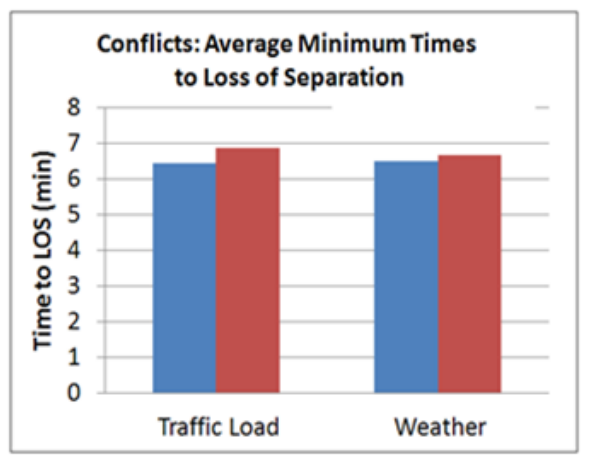

Figure 10: Time to conflict at resolution for each conflict pair

\section{Test-sector weather penetration}

One of the ANSP responsibilities in this simulation was to reroute aircraft as needed to avoid penetrating weather. Simulation pilots did not independently initiate or request weather avoidance maneuvers, so successful weather avoidance was solely attributable to ANSP actions.

The cumulative within-condition count of aircraft penetrating convective weather was greatly reduced in both test conditions when compared to the Open Loop results, with better performance observed in the MSP condition. Chi-square analyses were conducted on the aggregate counts for the four Weather scenario runs and showed that the MSP condition had significantly fewer weather penetrations $\left(\chi^{2}=6.33, p<0.01\right)$ (Figure 11). Analyses of the duration of weather penetration events showed a similar outcome. 


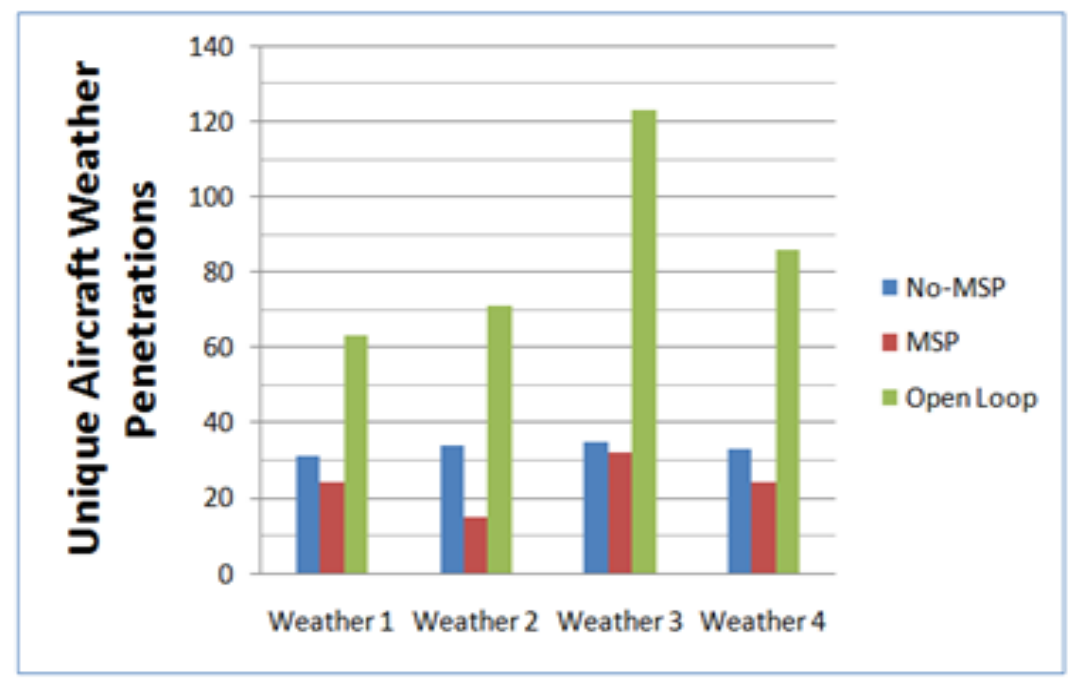

Figure 11: Weather penetration

\section{Flight efficiency}

Changes in flight path distance were determined for each aircraft that received one or more clearances and flew through, or was rerouted around, the ZKC East test sectors. Between-condition difference in mean path-length change for all Traffic Load scenarios was not significant when compared using a paired t-test $\left(\mathrm{M}_{\mathrm{MSP}}=+3.93 \mathrm{nmi}\right.$, $\mathrm{M}_{\text {NO-MSP }}=+3.49 \mathrm{nmi}$; $\mathrm{T}(3)=-0.98, p>0.39$ ). Average increases in path length observed during the Weather scenarios were also not significantly different between conditions $\left(\mathrm{M}_{\mathrm{MSP}}=+25.23 \mathrm{nmi}, \mathrm{M}_{\mathrm{NO}-\mathrm{MSP}}=+27.30 \mathrm{nmi} ; \mathrm{T}(3)\right.$ $=1.12, p>0.34$ ).

\section{B. Process Metrics}

This section analyzes between-condition differences in the distribution of clearance development, planning and communication activities among the ANSP team. These include differences in the development of lateral clearances during weather scenarios, sector aircraft count management in traffic load scenarios, and planning team communications.

\section{Lateral clearance differences during weather scenarios}

Open Loop results for each weather scenario were examined, and a total of 341 aircraft were identified that penetrated convective weather in one of the ZKC test sectors. Without a lateral weather avoidance maneuver, these same "Wx Aircraft" would also penetrate weather in the corresponding test runs. This section analyzes results for all Wx Aircraft that received lateral route uplink clearances (or "Lateral UCs") during a weather scenario test run. (see Figure 12).

The cumulative total number of Wx Aircraft that received lateral clearances during weather runs in the No-MSP condition $\left(\mathrm{T}_{\mathrm{No}-\mathrm{MSP}}=298\right)$ was not significantly different from the cumulative total in the MSP condition $\left(\mathrm{T}_{\mathrm{MSP}}=289 ; \chi^{2}(1)=0.14\right.$, $\mathrm{p}>0.71)$. However, the number of these aircraft that penetrated weather in the No-MSP condition $\left(\mathrm{T}_{\mathrm{No}-\mathrm{MSP}}=81\right)$ was significantly greater than in the MSP condition $\left(\mathrm{T}_{\mathrm{MSP}}=51 ; \chi^{2}(1)\right.$ $=6.82, \mathrm{p}<0.009)$. As Figure 12 shows, an interaction between condition and outcome was also observed $\left(\chi^{2}(1)=7.65, p<\right.$ 0.006).

Additional data indicate that the improved outcome in the MSP condition was not simply because more operators were sending more clearances, but that they were developing better solutions. The total count of Lateral UCs sent to Wx Aircraft for all weather runs was actually lower in the MSP condition ( $\mathrm{T}_{\mathrm{MSP}}$ $=488)$ than in the No-MSP condition $\left(\mathrm{T}_{\mathrm{No}-\mathrm{MSP}}=525\right)$, although

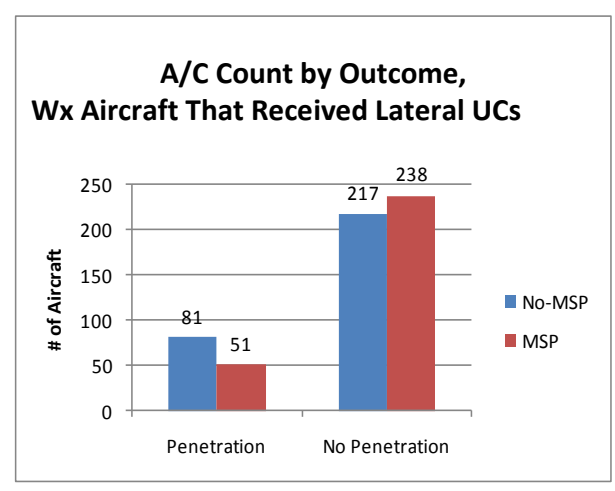

Figure 12: Wx Aircraft receiving lateral UCs that did and did not penetrate weather 
the difference was not significant $\left(\chi^{2}(1)=1.35, \mathrm{p}>0.24\right)$.

The bar charts in Figure 13 show a redistribution of clearance activity that may have contributed to this improved performance. The plot shows the number of Lateral UCs that were "initiated" from each of 3 positions: the test controller of the weather-impacted sector, the test controller of an upstream test sector, or a planner position (MSP or TMC). In the third case the trajectory clearance was either sent directly to the aircraft or coordinated through a Ghost controller working upstream from the test airspace.
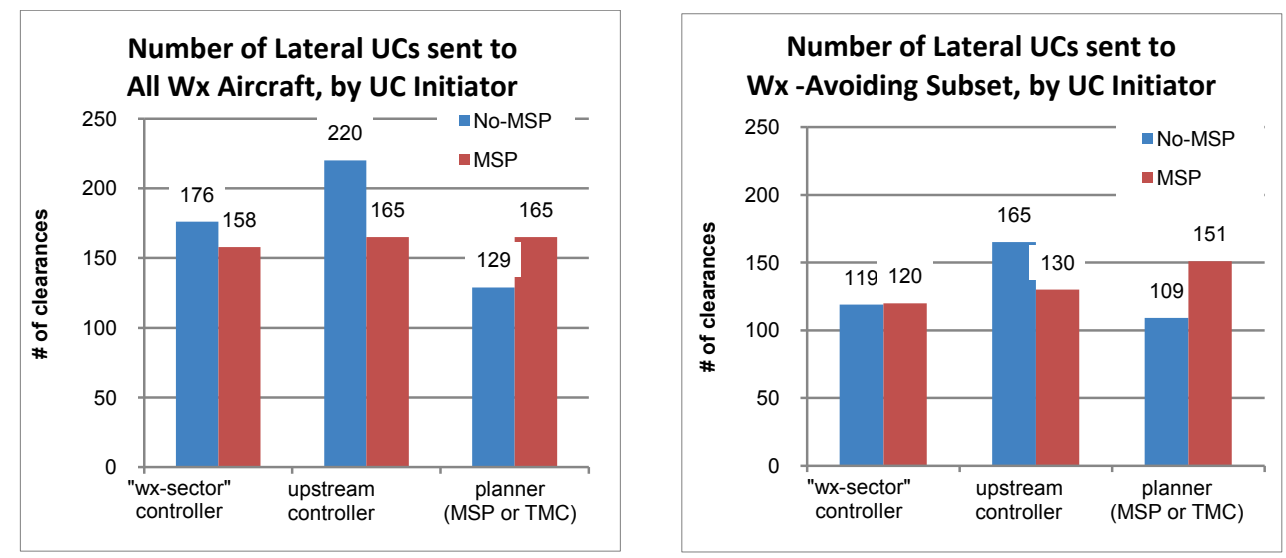

Figure 13: Number of lateral UCs initiated from the weather-affected test sector, an upstream test sector, or a planner station, to all Wx Aircraft (left), and initiator for weather-avoiding subset (right)

A shift towards more strategic delivery of weather avoidance clearances can be observed in the MSP condition, with an increase in the number of lateral clearances developed from MSP or TMC planning stations $\left(\mathrm{T}_{\mathrm{MSP}}=165\right.$, $\left.\mathrm{T}_{\mathrm{No}-\mathrm{MSP}}=129 ; \chi^{2}(1)=4.41, \mathrm{p}<0.04\right)$. More of those clearances were associated with successful weather avoidance (151 out of 165 for MSP condition, compared to 109 of 129 for No-MSP condition; $\chi^{2}(1)=3.49, p<0.07$ ). Probably as a direct consequence of this increase in effective planning activity, fewer lateral clearances were sent to the Wx Aircraft by test sector controllers $\left(\mathrm{T}_{\mathrm{MSP}}=323\right.$ vs. $\left.\mathrm{T}_{\mathrm{No}-\mathrm{MSP}}=396 ; \chi^{2}(1)=7.41, \mathrm{p}<0.007\right)$. It's likely that their reduced task load enabled these controllers to develop better clearances.

\section{Traffic Load Management}

A prediction of the number of aircraft exceeding a sector count threshold value for each of the test sectors was computed and summed across all seven sectors for 5 different time horizons: $60,45,30,15$ and 1 minute into the future. These excess aircraft predictions were updated at 1 minute intervals, and cumulative totals were computed for each 75minute run at each time horizon. Figure 14 presents aggregated totals for all of the four MSP and four No-MSP traffic load runs. Open Loop values were also determined and compared to the test results (Table 1).

The MSP condition had lower cumulative values for number of aircraft exceeding sectorcount thresholds than the No-MSP condition across all five prediction times. Moreover, Chisquare tests show significant reduction in predicted overload in the MSP condition compared to the No-MSP condition in all but the 45-min prediction time (bolded values, Table 1).

In order to examine if TMCs, MSPs, and supervisors/controllers were able to reduce

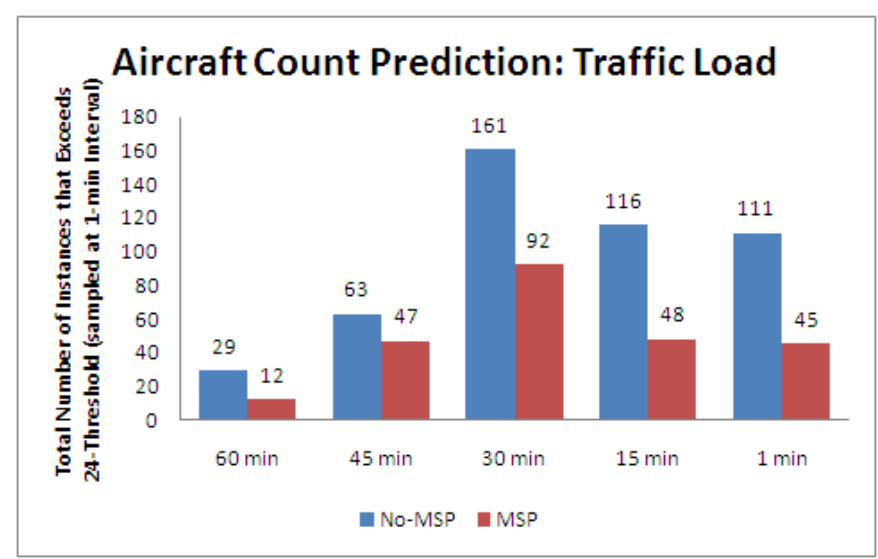

Figure 14: Aggregated totals of the excess number of aircraft within the test airspace

Table 1. Overload Predictions for all 4 Traffic Load Scenarios during MSP, No-MSP and Open Loop Runs

\begin{tabular}{|c|c|c|c|c|c|}
\hline $\begin{array}{l}\text { Predicted } \\
\text { Overload }\end{array}$ & $\begin{array}{c}60 \\
\min \\
\end{array}$ & $45 \mathrm{~min}$ & $30 \mathrm{~min}$ & $15 \mathrm{~min}$ & $1 \mathrm{~min}$ \\
\hline No-MSP & 29 & 63 & 161 & 116 & 111 \\
\hline MSP & 12 & 47 & 92 & 48 & 45 \\
\hline$\chi^{2}$ & $7.05^{* *}$ & 2.33 & $18.8^{* * *}$ & $26.3^{* * *}$ & $26.3^{* * *}$ \\
\hline Open Loop & 35 & 92 & 232 & 308 & 352 \\
\hline
\end{tabular}

9 
predicted overload at different time horizons, overload values in both test conditions were compared to the Open Loop values.

In the MSP condition, the results show that the TMC effectively reduced the aggregated excess aircraft values at 60 minutes $\left(\mathrm{N}=12\right.$ vs. 35 in Open Loop; $\left.\chi^{2}(1)=11.3, \mathrm{p}<0.001\right)$ and at 45 minutes $(\mathrm{N}=47$ vs. 92 in Open Loop; $\left.\chi^{2}(1)=14.6, p<0.001\right)$. The TMC was not as successful in reducing over-threshold values at 60 minute $(\mathrm{N}=29 \mathrm{vs}$. 35 in Open Loop; $\left.\chi^{2}(1)=0.56, p>0.4\right)$ in the No-MSP condition, but was able to reduce the number at 45 minute $\left(\mathrm{N}=63\right.$ vs. 92 in Open Loop; $\left.\chi^{2}(1)=5.43, \mathrm{p}<0.02\right)$. Sector load predictions were reduced in the MSP condition compared to the No-MSP condition, with significantly fewer instances of sector overload reported at the 60 and 30 minute prediction times. This suggests that in the MSP condition the TMC was able to concentrate on solving the traffic problem 60+ minutes into the future, while the MSPs effectively worked the 30-45 minute problem. Lack of significance in the $45 \mathrm{~min}$ prediction suggests that TMC actions in the No-MSP condition are similar to the MSPs actions in this timeframe.

\section{Communication and coordination}

There were a number of between-condition differences observed in the use of verbal and data communications for multi-sector planning by TMCs, supervisor and MSPs, and in coordinating clearance execution with controllers. ${ }^{12}$ Most planning and coordination between the TMCs and the supervisors was done verbally. In the MSP condition, data communications were used more often, to send trajectory modifications between MSPs and TMCs during the planning stages, and between MSPs and controllers during the plan implementation.

For the voice communication, the addition of the MSP position increased the frequency and complexity of communication and coordination activity. One obvious reason for increased communication frequency and coordination complexity is that the MSP condition included four additional people staffing the MSP positions compared to No-MSP condition. Figure 15 illustrates the increased communication frequency. As shown in Figure 15, the average number of calls made between two parties in the No-MSP condition was lower $(\mathrm{M}=34.5)$ than in the MSP condition $(\mathrm{M}=92.1)$. The data from multi-party conference calls followed a similar trend but there were very few during the study. The increased number of calls was both due to the additional communication initiated by the four additional MSP participants as well as the overall increase in call frequencies for the TMCs and supervisors due to their additional communications with the

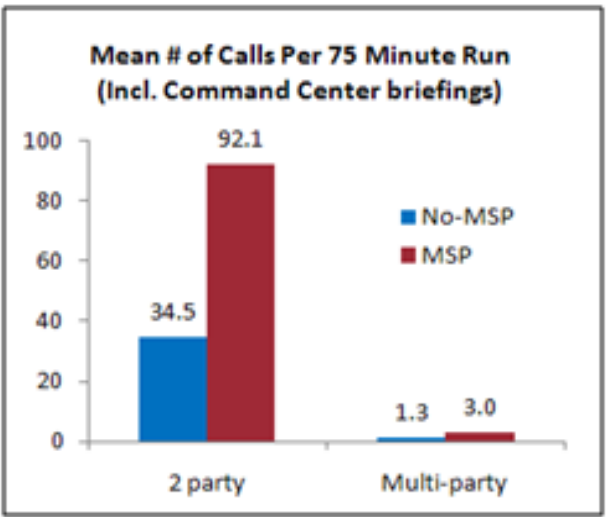

Figure 15: Average number of 2-party and multi-party planning calls per run for MSP and No-MSP condition MSPs.

The addition of the MSP position also increased the complexity of the coordination. In the No-MSP condition, the coordination between the TMCs and the supervisors was straightforward. In Figure 16, the left top and bottom figures (with blue arrows) represent the voice communication patterns from/to ZKC TMC and ZKC East supervisor, respectively. The arrows indicate the direction of the calls and the thickness of the arrows indicates the volume of the calls. As shown in the top left figure, ZKC TMC's voice communication patterns consisted mainly of coordinating flow plans with TMC Ghost who represented all TMCs from the adjacent Centers. The remaining calls by the ZKC TMC were to the ZKC East supervisor and Ghost supervisor who acted as the supervisor for the ZKC West airspace. The coordination between the ZKC TMC and the supervisors was for the implementation of trajectory/flow plans. Similarly, ZKC East supervisor had straightforward communication patterns with his own TMC (ZKC TMC) and the supervisor from the adjacent Area (Ghost supervisor acting as ZKC- West area supervisor).

In the MSP-condition, the communication patterns become much more complex with the addition of four MSP participants, as shown in the right top and bottom figures (with red arrows) in Figure 16. For the ZKC TMC, the top right figure shows that ZKC TMC still coordinates heavily with TMC Ghost ("other"), but his communications with the supervisors were reduced. The communication with ZKC TMC shifted instead to the MSPs in ZKC, presumably because in the MSP-condition, MSPs play the role of plan implementation that was performed by the supervisors in the No-MSP condition. Since MSPs are also plan initiators on their own right, there are a substantial number of calls that originate from the MSPs to ZKC TMC in ways not seen from the supervisors to the TMC in the No-MSP condition, resulting in many more calls coming into the ZKC TMC from more positions. 

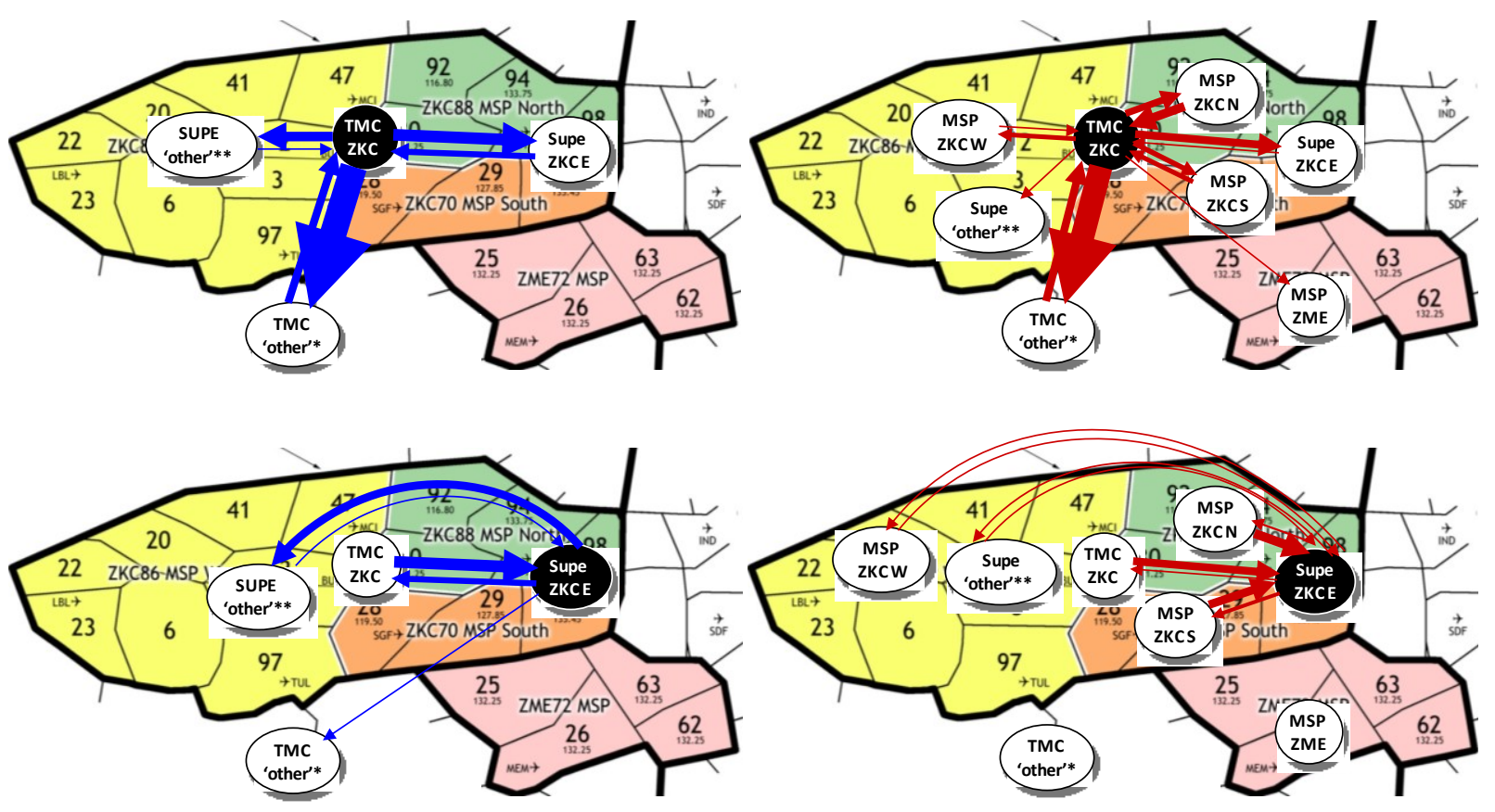

Figure 16: Voice communications between ZKC TMC (top)/ZKC East supervisor (bottom) and other positions in the No-MSP (left) and MSP (right) conditions. Outgoing calls are represented by arrows pointing away from ZKC TMC/ZKC East supervisor, incoming calls by arrows pointing to ZKC TMC/ZKC East supervisor. Relative frequency of calls is depicted by relative thickness of the arrows.

A closer look at how ZKC TMC voice communications changed between conditions illuminates the major differences in verbal coordination when MSPs are added to the team. The total number of calls placed and received by ZKC TMC was essentially unchanged between conditions, however the distribution changed for everything but TMC-to-TMC inter-facility coordination. Specifically, communications between ZKC TMC and ZKC East supervisor reduced by about half and communications between ZKC TMC and Ghost supervisor (acting as ZKC West supervisor) almost disappeared in the MSP condition. These calls were replaced by calls to (and from) the three ZKC MSPs, who took charge of the external coordination for approval seeking and plan execution.

Communications for ZKC East supervisor in the MSP condition show the same reduction in coordination with other supervisors as did ZKC TMC (Figure 16, bottom); the external coordination was shifted to the MSPs. Again, the communications between ZKC East supervisor and ZKC TMC communications are offloaded to ZKC MSPs North and South. Additionally, ZKC East supervisor receives more than twice as many calls in the MSP condition as in the No-MSP condition, with all additional calls coming from those two local MSPs. As expected, there were very few calls with MSP West, who coordinated instead with Ghost supervisor (acting as ZKC West supervisor).

In addition to the communication patterns shown in Figure 16, the MSP condition also had a substantial number of calls originating from one MSP to another, resulting in further complexity in the overall communication patterns. The added complexity seemed to be mainly due to a natural outcome of adding more ANSPs in the system but the impact seemed to have been magnified in the study by isolating each participant from the other and forcing them to communicate solely by using VCS. The isolation was a part of the study to examine the non co-location of MSPs from other team members which seemed feasible in a prior study ${ }^{7,8}$. In this study, however, the non co-location seemed to have added both to the number and the complexity of the coordination by forcing MSPs, TMCs, and Sups to put all impacted parties on the conference calls and share trajectory plans using ground-ground Data Comm. An alternative team configuration with co-location that puts TMC and all MSPs within a Center in the same room would significantly reduce the communication overhead and allow the TMC to be the central coordinator of the traffic/flow plans without overloading him.

Although the overall number of calls and the complexity of the coordination increased in the MSP condition, they were successfully and appropriately divided among TMCs, supervisors, and MSPs. Participants generally rated communication/coordination to be "easy". MSPs and supervisors rated coordination with various other team members to be between 1 and 2 on a rating scale of 1 (Very Easy) to 5 (Very Difficult). ZKC TMC rated the 
coordination with ZKC East supervisor to be easy (1.4) but rated the coordination with MSPs and TMC Ghost to be more difficult (2.8 and 2.3, respectively).

Despite the fact that the number of voice calls increased in the MSP condition, call durations were shorter in the MSP $(\mathrm{M}=35.9 \mathrm{sec})$ compared to the No-MSP condition $(\mathrm{M}=47.9 ; F(1,12)=7.73, p<0.05)$, as shown in Figure 17. The decrease in call duration offset the slight increase in the number of calls for ZKC TMC and ZKC East supervisor such that the number of total minutes that they spent on voice calls was comparable in the two conditions (see Figure 18). Furthermore, the figure also shows the proportion of time that the participants spent on voice calls of the full 75 minute runs were less than 22\% for the MSPs and 15\% for ZKC East supervisor, suggesting that they had enough time for other tasks such as traffic monitoring, flow planning, etc. ZKC TMC, on the other hand, spent approximately $40 \%$ of his time on voice calls, which may not have allowed him enough time for him to perform his other tasks.

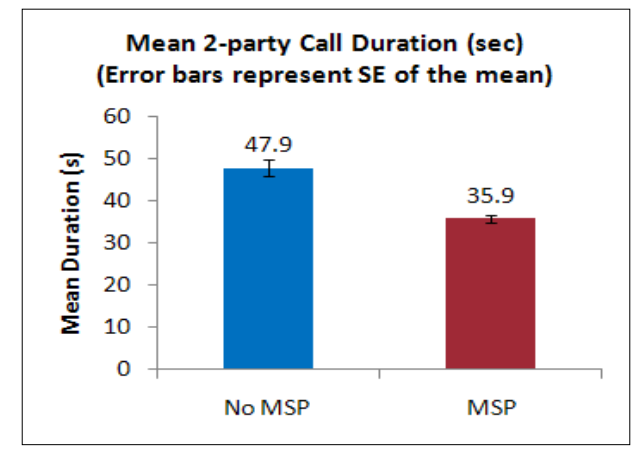

Figure 17: Mean duration of individual 2-party calls (in seconds) by condition

\begin{tabular}{|c|c|c|c|c|}
\hline \multicolumn{3}{|c|}{ Mean \# of Minutes on Voice Calls Per 75 Minute Run } \\
\hline & \multicolumn{5}{|c|}{ Position } \\
\hline Condition & TMC ZKC & $\begin{array}{c}\text { Area Sup } \\
\text { ZKC }\end{array}$ & $\begin{array}{c}\text { MSP ZKC } \\
\text { North }\end{array}$ & $\begin{array}{c}\text { MSP ZKC } \\
\text { South }\end{array}$ \\
\hline No MSP & 27.4 & 11.0 & & \\
\hline MSP & 30.2 & 10.7 & 16.4 & 16.4 \\
\hline
\end{tabular}

Figure 18: Average cumulative minutes of calls per 75 minute simulation runs by condition and position

\section{Operator Feedback: Acceptability, Scope and Location of Multi-Sector Planning Operations}

On the final day of each session, participant feedback was obtained through debrief discussions and post simulation questionnaires. The participants commented on various topics, including concept feasibility, communication/coordination, the role, location, and skill set of the MSPs, as well as other topics. Following is a high level summary of their feedback.

\section{Background/skills for MSP}

There was general consensus among participants regarding experience/skills/knowledge requirements for effective multi-sector planning. They felt that the person who is performing these functions would need controller skills, TMU experience, and good local area knowledge. They felt that TMU experience (or equivalent training) would be needed for the person to have a large system perspective on managing the overall traffic flows without being too protective of any given airspace. They thought that local area knowledge was crucial because in order to construct flow plans and to know where to reroute the aircraft, one needed to understand where the local airports, waypoints, and other geographic constraints were, as well as knowing the typical weather and wind patterns of the region. Finally, they felt that trajectory modification by a non-controller position would only be acceptable to the controllers if the routes looked "reasonable" and the person had the trust and credibility of the controllers who needed to review and accept the route modifications before sending them to the flight deck.

Overall, the controller participants commented that they were less concerned about who developed these trajectories than they were about quality of the trajectories. They found the idea of someone else (MSP or TMC) developing and proposing strategic clearances for them to execute was acceptable as long as they were credible. Controllers would have liked information about the objective of a clearance request, and more flexibility in its execution when possible. 


\section{MSP responsibilities and physical location}

The participants' descriptions of MSP responsibilities varied, as did their suggestions for how the role (or function) should be developed. Some MSP participants described how the position or tools might be used within the TMU, under the supervision of an STMC or TMCIC. Others focused on how the MSP supported area operations, referring to the position as a local-area monitor alert function, and local executor of TMU requests, and favored area collocation to facilitate effective MSP-Supervisor and even MSP-Controller interaction.

The main consideration with respect to physical location of the MSPs was the need for effective communication with other key players:

- MSP-MSP collocation would enable non-verbal coordination, improve collaboration for shared problem solving, and minimize chances for overlap or confusion of responsibilities.

- Locating the MSP in the Traffic Management Unit was described as better for top-down coordination. TMC management of MSP operations from a remote location was cumbersome for both teams. Participants indicated that more structure / more formal procedures would be needed if not co-located, resulting in more overhead and less flexible operations. Collocating MSPs in the TMU would also facilitate assignment to the MSP of monitor alert responsibility, allowing the TMC / STMC to be more system-focused.

- Locating the MSP in the area would greatly simplify MSP-supervisor coordination, and enable direct communication with controllers as appropriate. One Team 2 participant described how MSPs working directly in area would solve the problem of cumbersome verbal coordination between MSP-to-supervisor then supervisor-to-controllers. He suggested that the MSP could coordinate trajectories or particular solutions directly with controllers, with the supervisor informed but no longer a communication pathway / potential bottleneck. The supervisor would oversee the general plan but not need to mediate details of the implementation. A second benefit of area proximity is that MSPs can see who's busy, and which controllers need help, or are available to help, with plan execution.

Another reported advantage of area collocation was to facilitate trust. Controllers need to trust that there is a good reason for each MSP request even when the intent is not apparent, the supervisor needs to trust that the MSP is supporting his team's needs, and the MSP needs to trust that his requests will be addressed. While there were other suggestions for improving trust among team members (cross-training, position rotation, local area certification, intent communication), there was general consensus that increased proximity or visibility would help.

The participants varied responses suggest that there may be more than one way to integrate MSP functions into air traffic operations. They commonly described the MSP as a position, with particular skills and communication channels. However, when they were asked during the debrief discussion: "Is there any operation benefit to this position?" (as opposed to functional integration within the current team framework), there were no concerns raised about the latter solution.

\section{Communication and coordination}

Requiring all verbal coordination be carried out via the voice communication system highlighted the limitations of remote voice coordination, though it also highlighted the importance of good coordination among the distributed problem-solving team. Two potential voice coordination 'bottlenecks' with remotely located MSPs were reported by participants: (1) the ZKC TMC, as high level coordinator of MSP activities, and (2) ZKC area supervisor as the mediator of MSP-controller communications.

While participants suggested collocation of MSPs with their coordination partner as one solution, they also suggested that the communications could be streamlined or simplified. They thought the verbal communications were often more detailed than necessary, e.g., including specific aircraft and routes when more succinct general descriptions would have been sufficient.

Controllers suggested that additions or changes to the coordinated clearance request format could make it easier for the controller to make minor changes to the clearance while still satisfying the objective of the request. The suggested a simple addition to the message content indicating the underlying reason might sufficient. For example, adding "Wx" or "traffic" to the message might been satisfactory indicators for the weather avoidance and downstream congestion problems that were the two main drivers in our simulation.

In summary, there was overall consensus among participants about concept acceptability and feasibility, while the different suggestions for MSP problem scope and location indicate that there may be more than one best way to effectively integrate these functions into air traffic operations, and that they are likely to depend upon individual facility operations and constraints. 


\section{Discussion}

The results described in this report have been divided into analyses of outcome metrics, process metrics, communication/coordination data, and participant feedback. The outcome metrics measured how well the MSP condition with four added MSPs provided benefits in workload, safety, and efficiency. The overall results suggest that there were modest benefits in workload and safety but not in efficiency. In order to attribute the benefits to the MSP positions and their specific roles in the study, we examined how the benefits varied across various temporal and spatial horizons. In particular, since we defined the MSP positions to provide benefits by modifying trajectories from 30 to 60 minutes before the aircraft reaches the impacted sectors, we looked for benefits within these time and spatial horizons. The data suggest that the MSP condition provided lower predicted traffic overload and lower predicted weather penetrations at these horizons compared to the No-MSP condition. However, the impact of the benefits lessened once the aircraft entered the impacted sectors, presumably because supervisors/controllers were working on the problems in the near-term and within their own Area. Similarly the difference between the conditions were muted prior to these time horizons, presumably because TMC was working on the problems during the longer time horizons. Overall, the data suggest that the MSPs improved performance in their prescribed time horizons, and that the TMCs, supervisors, and controllers also produced similar benefits within their prescribed temporal and spatial horizons.

Communication and coordination processes were identified as a potential feasibility issue with MSP introduction due to a predicted increase in the frequency and complexity of communications. The overall number of voice calls increased in the MSP condition but the increase was mainly due to the added MSP participants. In fact, the mean total time per run involved in voice communications for the TMC and supervisor participants was similar across conditions due to a reduction in call length that offset the increase in the call frequency. Communication patterns with the inclusion of the MSP positions were more complex and they seemed to overload the TMC who had to act as the central coordinator. It seemed, however, that the added complexity could be significantly mitigated if the MSPs were co-located along with the TMC so that they could coordinate more quickly and easily.

Some of the comments from the participants supported the above findings and provided insights into the pros and cons of MSP positions as well as the efficacy of the trajectory modification functions from non-controller positions provided by the MSP tools. In particular, they commented that they really liked the MSP functions and tools but did not see the need for creating a new position. They suggested various ways of incorporating the MSP functions, both in the TMU and on the control floor to handle different types of traffic situations. Their feedback complemented the outcome data which showed modest benefits that did not seem to warrant the overhead of having new positions and the process data which suggested that TMCs and supervisors/controllers were performing similar functions as the MSPs but at different time horizons. Furthermore, communication/coordination data suggested that MSP positions added to the overall complexity of the coordination but the complexity could be managed if MSPs were co-located with each other and with other team members. Exploring the no-MSP option further, participants suggested moving the MSP positions to the TMU and converting them into TMC positions that are centrally coordinated by the Supervisor TMC (STMC). Others suggested moving them into the Area and embedding them into the control team. Overall, it seemed that the MSP functions would be best if they were distributed to existing team members who could use them differently for different situations within appropriate planning and implementation horizons.

\section{Conclusions}

A concept for multi-sector trajectory planning operations was developed for NextGen Mid-Term high altitude airspace. A key research question was whether these operations could be integrated into the roles and responsibilities of today's ANSP team positions, or would require a new staffed position: the multi-sector planner. A HITL simulation was designed and conducted to evaluate the multi-sector planning concept and its staffing requirements.

Simulation results showed effective multi-sector planning operations in both conditions, with test sector complexity and task load reduced to a manageable level. Although several performance measures-workload, weather avoidance, conflicts, sector complexity and load-were consistently better in the MSP condition, the observed differences were modest, and results were satisfactory in both conditions. These results combined with participant feedback indicate that the MSP functions are useful, but might be successfully integrated into the current ANSP team configuration, and may not require the introduction of a new MSP position.

\section{Future Work}

This simulation explored the use of multi-sector planning operations to manage problems caused by congestive weather and traffic congestion in en route airspace where all aircraft were equipped for air-ground data 
communications. Two recently-completed simulations investigated the use of multi-sector planning operations in other contexts. The first of these explored concept feasibility and requirements for mixed equipage airspace, with varying ratios of data communications equipped to unequipped air craft. The second simulation investigated use of multi-sector planning operations as a complement to flexible airspace management for matching traffic demand with capacity. In both simulations, multi-sector planning operations were integrated successfully into the traffic management and area supervisor roles.

\section{Acknowledgements}

This work was sponsored by NASA's Airspace Systems Program, and the FAA's ATO Planning, Research \& Technology Development Office. The NASA researchers would like to thank our NASA and FAA sponsors for their interest and support. We relied on their expertise and insights in steering this research, and are grateful for their interest and assistance. We would also like to thank the extensive team of FAA subject matter experts, both active and retired, who helped us develop and evaluate this concept.

\section{References}

${ }^{1}$ Technology Pathways: Assessing the Integrated Plan for a Next Generation Air Transportation System, National Academies Press, 2005, Page 17. http://darwin.nap.edu/books/0309097339/html/17.html

${ }^{2}$ Eurocontrol (1997). Trajectory negotiation in a Multi-Sector Environment. (PHAR/EEC/PD3-3.1.3.2.5/SSR;01). Brussels, Belgium: Author.

${ }^{3}$ Eurocontrol (1998). En Route Multi-Sector Planning procedures. (PHARE/EHQ/PAT-7.5.2.1;1.1). Brussels, Belgium, Author.

${ }^{4}$ Booz Allen Hamilton (2004). Analysis report of the concept of use for en route modernization. McLean VA: Author.

${ }^{5}$ Celio, J., Bolczak, R., \& Viets, K. (2005) Future En Route Sector Operational Description, Revision 1. (MTR 04W0000010R01). McLean, VA: The MITRE Corporation.

${ }^{6}$ Eurocontrol (2005). Multi Executive Sector Real-time simulation. (CEATS SSRTS 4) Central European Air Traffic Services Programme, Research and Development Centre, Budapest.

${ }^{7}$ Corker K., P. Lee, T. Prevot, E. Guneratne, L. Martin, N. Smith, S. Verma, J. Homola \& J. Mercer (2006). Analysis of Multi-Sector Planner Concepts in U.S. Airspace, report to the FAA.

${ }^{8}$ Corker, K., D. Liang, P. U. Lee, \& T. .Prevot (2007) New Air Traffic Management Concepts Analysis Methodology: Application to a Multi-Sector Planner in US Airspace, Air Traffic Control Quarterly Vol 15 (4)

${ }^{9}$ Prevot, T., C. Brasil, M. Mainini (2010). Multi-Sector Planning Tools for Trajectory-Based Operations, submitted to the $10^{\text {th }}$ AIAA Aviation Technology, Integration, and Operations (ATIO) Conference, September 13-15, Fort Worth TX.

${ }^{10}$ Mercer J, Prevot T, Brasil C, Mainini M, Kupfer M, and Smith N. An integrated tool suite for en route radar controllers in NextGen. Proceedings of the 27th International Congress of the Aeronautical Sciences, Nice, France, 2010.

${ }^{11}$ Smith, N., T. Prevot, P. Lee, C. Brasil, J. Homola, A. Kessell, H. Lee., J. Mercer, (submitted). Multi-Sector Planner II Simulation Report, submitted to the FAA ATO Planning, Research \& Technology Development Office.

${ }^{12}$ Kessell, A., Lee, P., Cabrall, C., and Smith, N. Ground-Ground Data Communication-Assisted Verbal Communication for Multi-Sector Air Traffic Management. Proceeding of the 29th European Association for Aviation Psychology Conference, Budapest, Hungary, 2010. 